AIAA 2001-5039

\title{
A STREAMLINED APPROACH FOR THE PAYLOAD CUSTOMER IN IDENTIFYING PAYLOAD DESIGN REQUIREMENTS
}

\author{
Ladonna J. Miller \\ NASA JSC \\ Houston, Texas USA \\ Walter F. Schneider \\ NASA MSFC \\ Huntsville, Alabama USA \\ Dexer E. Johnson \\ NASA JSC \\ Houston, Texas USA \\ Lesa B. Roe \\ NASA JSC \\ Houston, Texas USA
}

\begin{abstract}
NASA payload developers from across various disciplines were asked to identify areas where process changes would simplify their task of developing and flying flight hardware.

Responses to this query included a central location for consistent hardware design requirements for middeck payloads. The multidisciplinary team assigned to review the numerous payload interface design documents is assessing the Space Shuttle middeck, the SPACEHAB Inc. locker, as well as the MultiPurpose Logistics Module (MPLM) and EXpedite the PRocessing of Experiments to Space Station (EXPRESS) rack design requirements for the payloads. They are comparing the multiple carriers and platform requirements and developing a matrix which illustrates the individual requirements, and where possible, the envelope that encompasses all of the possibilities. The matrix will be expanded to form an overall envelope that the payload developers will have the option to utilize when designing their payload's hardware. This will optimize the flexibility for payload hardware and ancillary items to be manifested on multiple carriers and platforms with minimal impact to the payload developer.
\end{abstract}

This paper is declared a work of the U.S. Government and is not subject to copyright protection in the United States.

\section{Background}

Every human-rated vehicle and platform used in the extreme environment of space is incredibly complex. They have been developed to meet specific goals such as exploration, research, and construction. Each vehicle is designed to optimize the technology available at that time. Therefore, the overall characteristics of the Space Shuttle, a twenty year old design with the overall goal of being a reusable vehicle, as compared to the newer design of the International Space Station (ISS), which has the overall goal of being a continuously orbiting platform, are remarkably different. These differences are throughout the entire vehicles including the crew compartment and module interiors, and even to the rack level. With this diversity, the envelope of acceptable physical and environmental interfaces are markedly varied. These variances are due to the interface constraints and limitations inherent to both the Shuttle and the ISS. The structural, thermal, EMI/EMC and acoustical characteristics for crew compartment of the Space Shuttle differ from those of the interior of the Space Station. These types of differences cause the complexity of hardware to significantly increase if the hardware must be verified for more than one environment. 


\section{Documentation Overview}

The Space Shuttle Program overall payload design requirements are documented in the Shuttle Orbiter/Cargo Standard Interface Control Document - ICD 2-19001. These requirements include pass/fail specifications such as environmental measures, e.g. electromagnetic interference and conductance, factors of safety and utilization for structural assessments, power constraints, and numerous other areas. The subset of requirements applicable to the habitable area of the Space Shuttle vehicles are documented in the Middeck Interface Definition Document - NSTS 21000-MDK-IDD. This is commonly referred to as the middeck IDD. The middeck IDD requirements were incorporated into the EXPRESS Rack requirements documen EXPRESS Rack Payloads IDD, SSP 52000 EXP-IDD. The EXPRESS IDD defines the requirements a payload developer must meet to be transported to the ISS and perform science in the EXPRESS Rack.

\section{$\underline{\text { Status }}$}

A team of Space Shuttle Program (SSP) and ISS Program (ISSP) personnel were tasked to review the processes that a payload customer experiences when they fly a payload on both the Shuttle and the ISS. The major objective of the team was to simplify the customer interface to the ISS and the Shuttle Programs. A specific focus team addressed the concern of multiple payload requirements specifications for the various platforms. This team, known as the Common Middeck/Subrack Payload Requirement Team, had a goal of developing a global payload requirements envelope for the Shuttle middeck, a SPACEHAB locker, an EXPRESS rack, and an EXPRESS transportation rack that transports payloads in the multi-purpose logistics module (MPLM). The team started off this challenging task by reviewing existing documentation and identifying the various requirements. The requirements were then categorized as to whether or not a common envelope could be defined applicable to each of the four platforms. The structural loads requirement is still in negotiation stages to determine whether a common envelope can be identified. The categorization included negotiations with each of the platform teams to determine the potential common envelope and whether this common envelope would satisfy the requirements of each of the platforms. The following table summarizes the categories. The determination on commonality was driven by the platform with the least flexibility. Table 2, at the end of this paper, is a matrix of all of the requirements listed above with the specifications for each platform. In most cases, the limiter that establishes the common subset is easily identified. For example, ascent and descent power is constrained by the power specifications for the Shuttle middeck area. The middeck

\begin{tabular}{|c|c|c|}
\hline Common & In Work & Cannot Make Common \\
\hline Power & Loads & Vacuum Exhaust \\
\hline Structural Attachment & & Commanding and Data Downlink \\
\hline Weight and Center of Gravity & & Video \\
\hline $\begin{array}{c}\text { Environmental Temperature and } \\
\text { Pressure }\end{array}$ & & \\
\hline EMI/EMC & & \\
\hline $\begin{array}{c}\text { Depressurization/Repressurization Services } \\
\text { Rate }\end{array}$ & & \\
\hline Acoustic Noise & & \\
\hline Fire Protection & & \\
\hline Materials & & \\
\hline
\end{tabular}

Table 1 Categorized Requirements 
provides $28+/-4$ VDC on 10 and 20 amp circuits with total available power at 400 watts (W). The SPACEHAB single module has additional DC circuits and a greater maximum total DC power available. Therefore, for ascent and descent power, the common accommodations between these two platforms is constrained by what can be provided by the middeck. An example where there is no commonality is the on-orbit vacuum exhaust. There is no vacuum exhaust capability in the Shuttle middeck, or the MPLM. Therefore, there is no commonality other than for the payload to not use on-orbit vacuum exhaust. Since this is not reasonable for many payloads, their design must take into account that they cannot have access to vacuum exhaust while their payload is located in the middeck or the MPLM.

\section{Rationale}

The common envelope is most useful to those payloads that will be transported to ISS by the Space Shuttle, transferred to ISS for on-orbit operations, and then returned on the Space Shuttle. In this scenario, the payload could be placed in the middeck, SPACEHAB single module, or the MPLM in an EXPRESS rack or EXPRESS transportation rack for ascent and descent. For on-orbit ISS operations, the payload is installed in an EXPRESS rack. While the payload designer may know the platforms their hardware will interface with at the beginning of the design phase, the dynamic nature of the manifests may change their ascent and descent platforms multiple times before the hardware actually flies. Also, on-orbit anomalies may require transport of the hardware on an unanticipated platform. The benefit of designing hardware to an overall envelope is that it will be compatible with all of the platforms and the payload team will be minimally impacted by manifest adjustments or contingency situations. The downside to designing to an overall envelope is the increase in constraints, which may decrease the operations flexibility of the hardware. The hardware will have to meet the most extreme conditions including the tightest power specifications and the largest swing in environmental temperature and pressure. Therefore, it is likely that the cost of the hardware and necessary resource accommodations such as mass will increase because of the additional design constraints. These tradeoffs will have to be made by each payload team. With the matrix identifying the requirements for each platform, the payload team will have a guide available for comparison so that they can make the best choice for their payload. The matrix shown here is not a requirements document, and the payload developer must be aware that they will be required to verify their hardware against an Interface Control Document (ICD) they establish with the integrator of the platform they choose to use to perform science.

\section{Summary}

Since the payload developers that will benefit most from a set of common requirements across platforms are EXPRESS users, the common requirements envelope is being incorporated in the EXPRESS rack IDD, SSP 52000-IDD-ERP. Payload developers performing science on the ISS design to the requirements in SSP 52000 IDD-ERP. This approach maximizes manifest opportunities for payload developers. The payload developer who plans on performing science on other platforms will have the option to design to a specific platform or to use SSP 52000-IDD-ERP for their design requirements. The advantage of using SSP 52000-IDD-ERP is the payload will be able to perform science on all platforms and minimize reworking verification products. As mentioned earlier, a unique ICD will be developed between the integrator of the platform and the payload developer based on the platform's requirements. The payload developer will have to compare the options and determine the best approach for their hardware, operations, and budget. 


\section{Acronyms}

DC

EXPRESS

EMI

ICD

IDD

ISS

ISSP

JSC

MPLM

MSFC

NASA

NSTS

SSP

USA

VDC

W direct current

EXpedite the PRocessing of

Experiments to Space

Station

electromagnetic

interference

Interface Control

Document

Interface Definition

Document

International Space

Station

International Space

Station Program

Lyndon B. Johnson

Space Center

Multi-Purpose Logistics

Module

George C. Marshall

Space Flight Center

National Aeronautics

and Space

Administration

National Space

Transportation System

Space Shuttle Program

United States of

America

volts direct current

watts 


\begin{tabular}{|c|c|c|c|c|c|c|}
\hline REQUIREMENTS & $\begin{array}{c}\text { MIDDECK } \\
\text { (NSTS 21000-MDK-IDD) }\end{array}$ & $\begin{array}{c}\text { SPACEHAB } \\
\text { SINGLE MODULE }\end{array}$ & EXPRESS/MPLM & $\begin{array}{c}\text { EXPRESS/ISS } \\
\text { (Station Operations) }\end{array}$ & NOTES & $\begin{array}{c}\text { COMMONALITY } \\
\text { ACCOMMODATION }\end{array}$ \\
\hline $\begin{array}{c}\text { POWER } \\
\text { Ascent/Descent }\end{array}$ & $\begin{array}{l}\text { DC Power: } \\
28 \pm 4 \text { VDC } \\
10 / 20 \text { Amp circuits } \\
\text { Total Available Power: } \\
400 W \text { max. total DC } \\
\text { power in MD due to } \\
\text { limited cooling available. } \\
\text { No AC available }\end{array}$ & $\begin{array}{c}\text { DC Power: } \\
28 \pm 4 \text { VDC } \\
\text { Rack EPSU DC Circuits - } \\
35 \mathrm{~A}, 15 \mathrm{~A}, 10 \mathrm{~A}, 5 \mathrm{~A} \text {. } \\
\text { Locker DC Circuits }-5 \mathrm{~A}, 3 \mathrm{~A} \\
\text { Total Available Power: } \\
690 \mathrm{~W} \text { max. total DC power in single } \\
\text { module due to limited cooling available. } \\
\text { No AC available }\end{array}$ & $\begin{array}{l}\text { No power available to } \\
\text { payloads. }\end{array}$ & & $\begin{array}{l}\text { Payload total power } \\
\text { available is for the entire } \\
\text { carrier. Experiment } \\
\text { allocation available is } \\
\text { dependent on the mission } \\
\text { complement. }\end{array}$ & $\begin{array}{l}\text { DC Power: } \\
28 \pm 4 \text { VDC } \\
\text { 10/20 Amp Circuits } \\
\text { 400W max. total DC power } \\
\text { due to limited cooling } \\
\text { available. } \\
\text { No AC available } \\
\text { MPLM - } \\
\text { No power }\end{array}$ \\
\hline $\begin{array}{l}\text { POWER } \\
\text { On-Orbit }\end{array}$ & $\begin{array}{l}\text { DC power } \\
28 \pm 4 \mathrm{VDC} \\
10 / 20 \text { Amp circuits } \\
\text { Total Available Power: } \\
1400 \mathrm{~W} \text { w/o new power } \\
\text { panel } \\
1900 \mathrm{~W} \text { with new power } \\
\text { panel } \\
\\
\\
\text { AC Power } \\
115 \pm 5 \text { V RMS, } 400 \mathrm{~Hz} \\
3 \text { phase, } 120^{\circ} \\
\text { displacement } \\
300 \mathrm{VA} \text {, total capability }\end{array}$ & 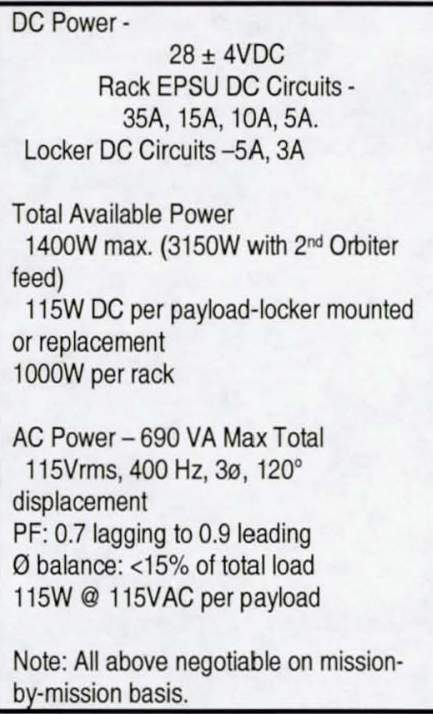 & $\begin{array}{l}\text { No power provided to } \\
\text { payloads. }\end{array}$ & $\begin{array}{c}\text { DC Power } \\
28+1.5 /-3.0 \mathrm{VDC} \\
5,10,15,20 \mathrm{~A} \text { services. } \\
\\
\text { Total Available Power: } \\
2000 \mathrm{~W} / \mathrm{rack} \text { max. (may be } \\
\text { limited by thermal cooling } \\
\text { restrictions) } \\
\text { Nominal power per payload } \\
\leq 150 \mathrm{~W} \text {. } \\
\text { Max. power per payload } \\
500 \mathrm{~W} \text {. } \\
\text { AC Power } \\
\text { Not available }\end{array}$ & & $\begin{array}{l}\text { DC Power: } \\
28+1 / 5 /-3.0 \mathrm{VDC} \\
\text { 10/20Amp circuits } \\
\text { No AC power } \\
\text { Total Available DC Power: } \\
\text { 1000W per rack with nominal } \\
\text { 115W per payload-locker } \\
\text { mounted or replacement. }\end{array}$ \\
\hline $\begin{array}{c}\text { STRUCTURAL } \\
\text { ATTACHMENT } \\
\text { All Phases }\end{array}$ & $\begin{array}{l}\text { Lockers } \\
\text { Soft Stowage Bags } \\
\text { Locker replacement } \\
\text { (Payload mounting } \\
\text { panels, single/double } \\
\text { adapter plates, vented } \\
\text { payload mounting panels) }\end{array}$ & $\begin{array}{l}\text { Lockers } \\
\text { Soft Stowage Bags } \\
\text { Locker replacement (Payload mounting } \\
\text { panels, single/double adapter plates, } \\
\text { vented payload mounting panels) } \\
\text { Two single or double racks. } \\
\text { One ISPR or EXPRESS rack. }\end{array}$ & $\begin{array}{l}\text { Lockers } \\
\text { ISIS Drawers } \\
\text { Locker replacement } \\
\text { (Payload mounting } \\
\text { panels, single/double } \\
\text { adapter plates, vented } \\
\text { payload mounting } \\
\text { panels) }\end{array}$ & & $\begin{array}{l}\text { SPACEHAB Currently } \\
\text { cannot accommodate ISIS } \\
\text { Drawers but can upgrade } \\
\text { rack to accommodate as } \\
\text { optional service. } \\
\text { VPMPs not currently } \\
\text { available. }\end{array}$ & $\begin{array}{l}\text { Lockers } \\
\text { Locker replacement }\end{array}$ \\
\hline
\end{tabular}




\begin{tabular}{|c|c|c|c|c|c|c|}
\hline REQUIREMENTS & $\begin{array}{c}\text { MIDDECK } \\
\text { (NSTS 21000-MDK-1DD) }\end{array}$ & $\begin{array}{c}\text { SPACEHAB } \\
\text { SINGLE MODULE }\end{array}$ & EXPRESS/MPLM & $\begin{array}{c}\text { EXPRESS/ISS } \\
\text { (Station } \\
\text { Operations) }\end{array}$ & NOTES & $\begin{array}{c}\text { COMMONALITY } \\
\text { ACCOMMODATION }\end{array}$ \\
\hline $\begin{array}{l}\text { WEIGHT \& C. G. } \\
\text { All Phases }\end{array}$ & $\begin{array}{l}\text { Locker: } \\
70 \text { lb @ } 10 \text { in. CG (PL, locker, } \\
\text { foam, trays, mtg hdwe) } \\
\text { Locker Replacement: } \\
\text { Single Plate: } 69 \mathrm{lb} @ 10 \mathrm{in} . \\
\text { CG (includes mtg. plate } \\
\text { weight) } \\
\text { Double Plate: } 120 \mathrm{lb} @ 10 \mathrm{in} . \\
\text { CG (includes mtg. plate } \\
\text { weight) }\end{array}$ & $\begin{array}{l}\text { Locker: } \\
76 \text { lb @ } 10 \text { in. CG (PL, locker, } \\
\text { foam, trays, mtg. hdwe) } \\
\text { Locker Replacement: } \\
\text { Single Plate: } 76 \text { lb @ } 10 \text { in. CG } \\
\text { (includes SH adapter plate wt.) } \\
\text { Double Plate: } 133 \text { lb @ } 14 \text { in. } \\
\text { CG (includes SH adapter plate wt.) }\end{array}$ & $\begin{array}{l}\text { Locker: } 72 \text { lb @ } 10 \text { in. CG } \\
\text { Locker replacement: } \\
\text { Single Plate: } 72 \text { lb @ } 10 \text { in. CG } \\
\text { Double Plate: } 140 \text { lb @ } 10 \text { in. CG } \\
\text { ISIS Drawer: } 64 \text { lb @ } 15.6 \text { in. CG } \\
\text { (incl. Drawer, slides, and PL) }\end{array}$ & & $\begin{array}{l}\text { *Weight depends on } \\
\text { stowage location; } \\
\text { max. shown for } 1.0 \\
\text { MLVE. }\end{array}$ & $\begin{array}{l}\text { Locker: } \\
70 \mathrm{lb} @ 10 \text { in. CG (PL, } \\
\text { locker, foam, trays, mtg. } \\
\text { hdwe) } \\
\text { Locker Replacement: } \\
\text { Single Plate: } 69 \mathrm{lb} @ 10 \text { in. } \\
\text { CG (includes mtg. plate } \\
\text { weight) } \\
\text { Double Plate: } 120 \mathrm{lb} @ 10 \text { in. } \\
\text { CG (includes mtg. Plate } \\
\text { weight) }\end{array}$ \\
\hline$\frac{\text { LOADS }}{\underline{\text { Ascent }}}$ & $\begin{array}{l}X=9.0 \mathrm{~g} \text { 's } \\
Y=3.2 \text { g's } \\
Z=7.4 \text { g's } \\
\text { Orbiter coordinates. } \\
\text { Random load factors } \\
\text { included. }\end{array}$ & 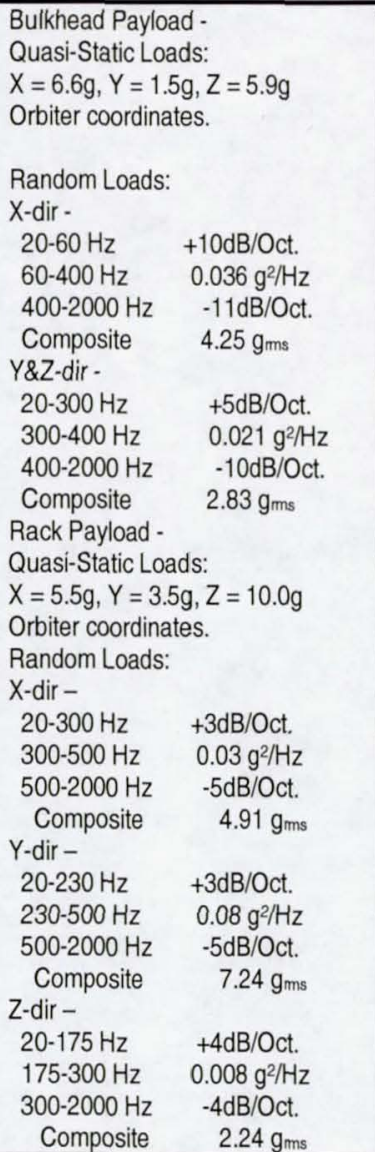 & 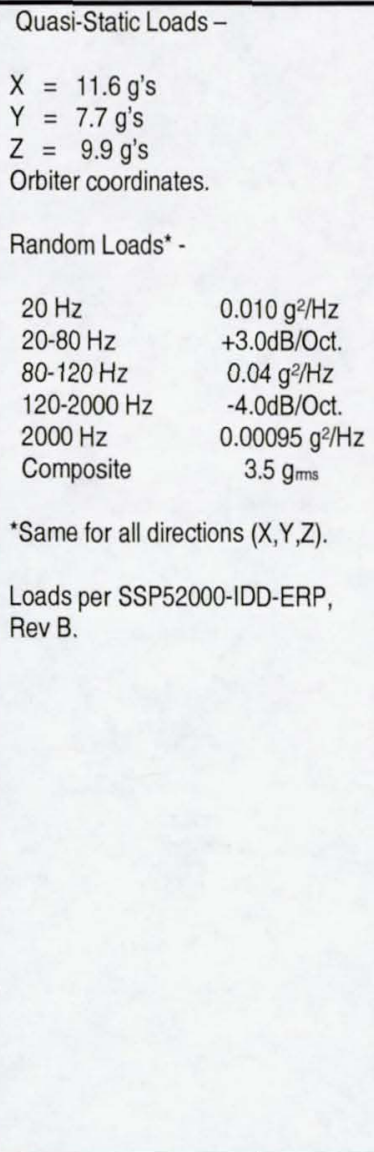 & & $\begin{array}{l}\text { Note: For } \\
\text { SPACEHAB and } \\
\text { MPLM, the quasi- } \\
\text { static and random } \\
\text { loading must be } \\
\text { combined for liftoff } \\
\text { conditions per } \\
\text { paragraph } 4.1 .2 \text { of } \\
\text { SSP } 52005 . \text { A } \\
\text { comparison can then } \\
\text { be made between } \\
\text { carriers to determine } \\
\text { which carrier has the } \\
\text { critical load factors. } \\
\text { Structures working } \\
\text { group will be } \\
\text { contacted for input } \\
\text { on commonality } \\
\text { requirement. }\end{array}$ & TBD \\
\hline
\end{tabular}




\begin{tabular}{|c|c|c|c|c|c|c|}
\hline REQUIREMENTS & $\begin{array}{c}\text { MIDDECK } \\
\text { (NSTS 21000MDK-DD) }\end{array}$ & $\begin{array}{c}\text { SPACEHAB } \\
\text { SINGLE MODULE }\end{array}$ & EXPRESS/MPLM & $\begin{array}{c}\text { EXPRESS/ISS } \\
\text { (Station Operations) }\end{array}$ & NOTES & $\begin{array}{c}\text { COMMONALITY } \\
\text { ACCOMMODATION }\end{array}$ \\
\hline $\begin{array}{l}\text { LOADS } \\
\text { Descent }\end{array}$ & $\begin{array}{l}X=6.25 \mathrm{~g} \text { 's } \\
Y=2.50 \mathrm{~g} / \mathrm{s} \\
Z=12.5 \mathrm{~g} \text { 's } \\
\text { Orbiter coordinates. }\end{array}$ & $\begin{array}{l}\text { Bulkhead Payloads } \\
X=9.5 \text { g's } \\
Y=2.50 \mathrm{~g} \text { 's } \\
Z=9.00 \mathrm{~g} \text { 's } \\
\text { Orbiter coordinates. } \\
\text { Rack Payloads } \\
X=6.5 \mathrm{~g} \text { 's } \\
Y=2.5 \mathrm{~g} \text { 's } \\
Z=12.5 \mathrm{~g} \text { 's } \\
\text { Orbiter coordinates. }\end{array}$ & $\begin{array}{l}X=9.5 \mathrm{~g} \text { 's } \\
Y=5.4 \mathrm{~g} \text { 's } \\
Z=12.5 \mathrm{~g} \text { 's } \\
\text { Orbiter coordinates. } \\
\text { Loads per SSP52000-IDD-ERP, } \\
\text { Rev B. }\end{array}$ & & $\begin{array}{l}\text { Note: Random loading } \\
\text { not applicable for descent } \\
\text { conditions. }\end{array}$ & TBD \\
\hline $\begin{array}{l}\text { ENVIRONMENTAL } \\
\text { TEMPERATURE } \\
\text { AND PRESSURE } \\
\text { Ascent/Descent }\end{array}$ & $\begin{array}{l}\text { Air Temp. } * \text { : } 65-80 \mathrm{~F} \\
\text { Dew Pt.: } 39-61 \mathrm{~F} \\
\text { Pressure: } 14.5-16.0 \text { psia } \\
\text { O2 max: } 25.9 \% \\
\text { CO2 PP: TBD }\end{array}$ & $\begin{array}{l}\text { Air Temp.: } 41-95 \mathrm{~F} \\
\text { Dew Pt.: } 41-63 \mathrm{~F} \\
\text { Pressure: } 14.5-15.95 \text { psia } \\
\text { O2 max: } 25.9 \% \\
\text { CO2 PP: } 7.6 \mathrm{mmHg}\end{array}$ & $\begin{array}{llc} & \text { ASCENT } & \text { DESCENT } \\
\text { Air Temp.: } & 57-75 \mathrm{~F} & 55-109 \mathrm{~F} \\
\text { Dew Pt.: } & 56.8 \mathrm{~F} & 50 \mathrm{~F} \\
\text { Pressure: } & 13.9-15.2 p s i a & 13.9- \\
& & 15.2 \mathrm{psia} \\
\text { O2 max: } & 24.1 \% & 24.1 \% \\
\text { CO2 PP: } & 7.5 \mathrm{mmHg} & 7.5 \mathrm{mmHg}\end{array}$ & & $\begin{array}{l}\text { *95F max peak possible } \\
\text { during contingency } \\
\text { operations. No time @ } \\
\text { Temp available. }\end{array}$ & $\begin{array}{l}\text { Air Temp.: } 41-109 \mathrm{~F} \\
\text { Dew Pt.: } 39-63 \mathrm{~F} \\
\text { Pressure: } 13.9-16.0 \\
\text { psia } \\
\text { O2 max: } 25.9 \% \\
\text { CO2 PP: } 7.5 \mathrm{mmHg} \\
\text { Includes MPLM. }\end{array}$ \\
\hline $\begin{array}{c}\text { ENVIRONMENTAL } \\
\text { TEMPERATURE } \\
\text { AND PRESSURE } \\
\text { On-Orbit }\end{array}$ & $\begin{array}{l}\text { Air Temp.: } 65-80 \mathrm{~F} \\
\text { Dew Pt.: } 39-61 \mathrm{~F} \\
\text { Pressure: } 14.5-16.0 \text { psia } \\
\text { O2 max: } 25.9 \% \\
\text { CO2 PP: TBD }\end{array}$ & $\begin{array}{l}\text { Air Temp: } 65-80 \mathrm{~F} \\
\text { Dew Pt.: } 41-63 \mathrm{~F} \\
\text { Press: *14.5-15.95 psia (nominal) } \\
9.7-15.95 \text { (worst case) } \\
\text { depending on EVA constraints. }\end{array}$ & $\begin{array}{l}\text { Air Temp: } 73-113 \mathrm{~F} \\
\text { Dew Pt.: } 60 \mathrm{~F} \\
\text { Press.: } 13.9-15.2 \text { psia }\end{array}$ & $\begin{array}{l}\text { Air Temp.: 63-82F } \\
\text { Dew Pt.: 40-60F } \\
\text { Press: } 14.2-14.9 \text { psia } \\
\text { (nominal) }\end{array}$ & ${ }^{*}$ (Control by orbiter) & $\begin{array}{l}\text { Air Temp.: } 65-113 \mathrm{~F} \\
\text { Dew Pt.: } 39-63 \mathrm{~F} \\
\text { Pressure: } 9.7-16.0 \\
\text { psia } \\
\text { O2 max: } 25.9 \% \\
\text { CO2 PP: TBD }\end{array}$ \\
\hline $\begin{array}{l}\text { EMI/EMC } \\
\text { All Phases }\end{array}$ & Comparable & Comparable & Comparable & Comparable & $\begin{array}{l}\text { Will consult EME panel } \\
\text { for commonality }\end{array}$ & $\mathrm{TBD}$ \\
\hline $\begin{array}{l}\text { PAYLOAD } \\
\text { THERMAL } \\
\text { CONTROL } \\
\text { Ascent/Descent }\end{array}$ & $\begin{array}{l}\text { Passive air cooling: } \\
\text { 60W max. per single } \\
\text { locker payload } \\
\text { Cabin discharge: } \\
\text { Front breathing, non- } \\
\text { ducted, PL provided fan, } \\
\text { max. air out temp. 120F. } \\
\text { Avionics Bay: } \\
\text { Ducted cooling, PL } \\
\text { provided fan, rear } \\
\text { breathing, discharge to } \\
\text { avionics bays } 1,2,3 \mathrm{~A} \text {. } \\
\text { 400W max. heat load } \\
\text { allowed in the cabin due to } \\
\text { cooling limitations. }\end{array}$ & $\begin{array}{l}\text { Passive air cooling: } \\
60 \mathrm{~W} \text { max. per single locker } \\
\text { payload. } \\
\qquad \text { Cabin discharge: } \\
\text { Front breathing, } 120 \mathrm{~F} \text { max. air } \\
\text { outlet, PD provides air circ. hdwe. } \\
\text { SH rack provided cooling via } \\
\text { RSCS } \\
\text { 690W total max. cooling for } \\
\text { payloads. } \\
105 \text { CFM max. air flow per rack. }\end{array}$ & $\begin{array}{l}\text { Not required - no power available } \\
\text { for payload operation. }\end{array}$ & & $\begin{array}{l}\text { EXPRESS rack and } \\
\text { Middeck lockers designed } \\
\text { for rear breathing forced } \\
\text { air cooling. } \\
\text { SPACEHAB can provide } \\
\text { rear breathing on a case } \\
\text { by case basis. } \\
\text { SPACEHAB can provide } \\
\text { water cooling via pump } \\
\text { package to both racks } \\
\text { and lockers } \\
\text { Middeck maximum } \\
\text { cooling capability is } \\
\text { different for each bay. } \\
\text { Refer to NSTS } 2100 \text {-IDD- } \\
\text { MDK, Tables } 6.2 .1 .5 .1 \\
\text { through } 6.2 .1 .5 .3 . \text { for } \\
\text { cooling capability for each } \\
\text { bay. }\end{array}$ & $\begin{array}{l}\text { Passive air cooling: } \\
60 \text { W max. per single } \\
\text { locker payload } \\
\\
\text { Cabin discharge: } \\
\text { Front breathing, non- } \\
\text { ducted, PL provided } \\
\text { fan, max. air out temp. } \\
120 \mathrm{~F} \text {. } \\
\text { 400W max. power for } \\
\text { all middeck items } \\
\text { allowed due to cooling } \\
\text { limitations. } \\
\text {.................. } \\
\text { MPLM- } \\
\text { No requirement, no } \\
\text { power. }\end{array}$ \\
\hline
\end{tabular}




\begin{tabular}{|c|c|c|c|c|c|c|}
\hline REQUIREMENTS & $\begin{array}{c}\text { MIDDECK } \\
\text { (NSTS 21000-MDK-1DO) }\end{array}$ & $\begin{array}{c}\text { SPACEHAB } \\
\text { SINGLE MODULE }\end{array}$ & EXPRESS/MPLM & $\begin{array}{c}\text { EXPRESS/ISS } \\
\text { (Station Operations) }\end{array}$ & NOTES & $\begin{array}{c}\text { COMMONALITY } \\
\text { ACCOMMODATION }\end{array}$ \\
\hline $\begin{array}{l}\text { PAYLOAD } \\
\text { THERMAL } \\
\text { CONTROL } \\
\text { On-Orbit }\end{array}$ & $\begin{array}{l}\text { Cabin discharge: } \\
\text { Non-ducted PL provided fan, } \\
\text { max. air out temp. } 120 \mathrm{~F} \text {. } \\
\text { Avionics Bay: } \\
\text { Ducted cooling, PL provided } \\
\text { fan, rear breathing, discharge } \\
\text { to avionics bays } 1,2,3 \mathrm{~A} \text {. } \\
1500-2000 \mathrm{~W} \text { max. heat load } \\
\text { to cabin. } \\
\ldots \\
\text {......................... } \\
\text { Passive cooling: } 60 \mathrm{~W} \text { max. } \\
\text { per single payload. }\end{array}$ & $\begin{array}{l}1400 \mathrm{~W} \text { surface air } \\
\text { cooling. } \\
2000 \text { W Rack suction } \\
\text { cooling } \\
60 \mathrm{~W} \text { per locker - } \\
\text { passive cooling. } \\
120 \mathrm{~F} \text { max. exhaust } \\
\text { air. temperature for } \\
\text { forced air cooling. }\end{array}$ & $\begin{array}{l}\text { Not applicable. No } \\
\text { power provided to } \\
\text { payloads. }\end{array}$ & $\begin{array}{l}\text { Avionics Air Assembly: } \\
15 \pm 3 C F M \text { total air available } \\
\text { per locker position. } \\
65-85 \mathrm{~F} \text { - inlet air } \\
120 \mathrm{~F} \text { max. - outlet air (Air } \\
\text { temp. can vary depending on } \\
\text { payload heat load). } \\
\text { Max. air cooling of } 1200 \mathrm{~W} \text { for } \\
\text { payloads. } \\
\text { Payloads to provide internal } \\
\text { fan for air flow. } \\
\text { Water Loop: } \\
1000 \mathrm{~W} \text { max. @ } 200 \mathrm{lbm} / \mathrm{hr} \\
\text { (Limited to } \leq 2 \text { payloads). } \\
61-73 \mathrm{~F}-\text { inlet water } \\
120 \mathrm{~F} \text { max. - outlet water. } \\
\\
\text { Combined payload cooling (air } \\
\text { and water) not to exceed } \\
2000 \mathrm{~W} \text { per rack. }\end{array}$ & $\begin{array}{l}\text { EXPRESS rack and Middeck } \\
\text { lockers designed for rear } \\
\text { breathing forced air cooling. } \\
\text { Rear breathing possible on SH } \\
\text { for lockers at the bulkhead; } \\
\text { selective placement required to } \\
\text { prevent warm air suction into } \\
\text { adjacent locker payloads. Max. } \\
\text { cooling in this configuration is } \\
\text { TBD. }\end{array}$ & $\begin{array}{l}\text { Max. air cooling: } 1200 \mathrm{~W} \text { fo } \\
\text { payloads. } \\
120 \mathrm{~F} \text { max. outlet air } \\
\text { temperature. } \\
\text { PL to provide internal fan. } \\
\text { Passive cooling: } 60 \mathrm{~W} \text { per } \\
\text { locker } \\
\text { MPLM: } \\
\text { No requirement, no power. }\end{array}$ \\
\hline $\begin{array}{c}\text { VACUUM } \\
\text { EXHAUST } \\
\text { Ascent/Descent } \\
\end{array}$ & Not available. & Not available. & Not available. & & & Not available. \\
\hline $\begin{array}{l}\text { VACUUM } \\
\text { EXHAUST } \\
\text { On-Orbit }\end{array}$ & Not available. & $\begin{array}{l}\text { One Experiment Vent } \\
\text { Value (EVV) Available } \\
\text { for Experiment Shared } \\
\text { usage. } \\
\text { System available to } \\
\text { locker or rack } \\
\text { payloads on shared, } \\
\text { time-lined basis. } \\
\text { Gases only; flow } \\
\text { control and isolation } \\
\text { provided by payload. }\end{array}$ & Not available. & $\begin{array}{l}\text { Available to each rack on } \\
\text { shared, time-lined basis. Gases } \\
\text { only; flow control and isolation } \\
\text { provided by payload. }\end{array}$ & & Not available. \\
\hline $\begin{array}{l}\text { COMMANDING } \\
\text { (uplink) } \\
\text { DATA (downlink) } \\
\text { Ascent/Descent }\end{array}$ & Not Available & Not Available & Not available & & & Not available. \\
\hline
\end{tabular}




\begin{tabular}{|c|c|c|c|c|c|c|}
\hline REQUIREMENTS & $\begin{array}{c}\text { MIDDECK } \\
\text { (NSTS 21000MOMK-1D0) }\end{array}$ & $\begin{array}{c}\text { SPACEHAB } \\
\text { SINGLE MODULE }\end{array}$ & EXPRESS/MPLM & $\begin{array}{c}\text { EXPRESS/ISS } \\
\text { (Station Operations) }\end{array}$ & NOTES & $\begin{array}{c}\text { COMMONALITY } \\
\text { ACCOMMODATION }\end{array}$ \\
\hline $\begin{array}{l}\text { COMMANDING } \\
\text { (uplink) } \\
\text { DATA (downlink) } \\
\text { On-Orbit }\end{array}$ & $\begin{array}{l}\text { Available from a } \\
\text { PGSC via the OCA } \\
\text { system. }\end{array}$ & $\begin{array}{l}\text { DMU provides serial, analog, and } \\
\text { discrete signal interfaces to the payload. } \\
\text { The total number of DMU inputs and } \\
\text { outputs available to a particular payload } \\
\text { will be determined on a mission by } \\
\text { mission basis. } \\
\text { Racks: } \\
16 \text { (DILs) } 12 \text { (DIHs) } 8 \text { (DOHs) } 32 \text { (Als) } \\
\text { also two serial DMMU channels are } \\
\text { available at each rack location. } \\
\text { Bulkhead mounted Payloads: } \\
72 \text { (DILs) } 56 \text { (DIHs) } 24 \text { (DOHs) } 104 \text { (Als) } \\
\text { and } 8 \text { serial DMU channels } \\
\text { RS232 via (serial converter unit) }\end{array}$ & None & $\begin{array}{c}\text { Interfaces per rack } \\
\text { payload:* } \\
\text { 1-RS422 } \\
\text { 1- Ethernet } \\
\text { 2-Analog** } \\
\text { 3- } \text { Discrete }^{* *}\end{array}$ & $\begin{array}{l}\text { All Spacehab payload inputloutput } \\
\text { data is via the Data Management } \\
\text { Unit (DMU) through bulkhead } \\
\text { mounted experiment connector } \\
\text { panels } \\
\text { * Data interfaces of the EXPRESS } \\
\text { rack RIC/SSPCM, the Middeck } \\
\text { system, and the Spacehab DMU } \\
\text { are not compatible. } \\
\text { * } \\
\text { "Routed through the SSPCM } \\
\text { "All locker mounted payload } \\
\text { inputloutput data as via the Data } \\
\text { Management Unit (DMU) through } \\
\text { bulkhead mounted experiment } \\
\text { connector panels in the Single } \\
\text { Module. The EDSMU could be } \\
\text { made available. Will state } \\
\text { requirement capabilities in the } \\
\text { RDM section later. } \\
\text { * DMU \& EXPRESS rack Rack } \\
\text { Interface Controller (RIC) interface } \\
\text { characteristics are not compatible. }\end{array}$ & TBD \\
\hline $\begin{array}{c}\text { VIDEO } \\
\text { Ascent/Descent }\end{array}$ & Not Available & Not Available & Not Available & Not Available & & Not available. \\
\hline $\begin{array}{l}\text { VIDEO } \\
\text { On-Orbit }\end{array}$ & $\begin{array}{l}\text { Standard video } \\
\text { downlink available. }\end{array}$ & $\begin{array}{l}\text { Video Switching Unit (VSU) } \\
8 \text { unbalanced (single ended) inputs. The } \\
\text { VSU provides one balanced output to } \\
\text { the Orbiter CCTV system and one } \\
\text { unbalanced output is available as an } \\
\text { input to a video digitizer/compressor } \\
\text { provided by SPACEHAB. Two aux.outs } \\
\text { for attaching LCD monitors. }\end{array}$ & None & $\begin{array}{l}1 \text { - Video (2 for ISIS } \\
\text { drawer payload) }\end{array}$ & & Not available. \\
\hline
\end{tabular}




\begin{tabular}{|c|c|c|c|c|c|c|}
\hline REQUIREMENTS & $\begin{array}{c}\text { MIDDECK } \\
\text { (NSTS 21000-MDK-1DD) }\end{array}$ & $\begin{array}{c}\text { SPACEHAB } \\
\text { SINGLE MODULE }\end{array}$ & EXPRESS/MPLM & $\begin{array}{c}\text { EXPRESS/ISS } \\
\text { (Station Operations) }\end{array}$ & NOTES & $\begin{array}{c}\text { COMMONALITY } \\
\text { ACCOMMODATION }\end{array}$ \\
\hline $\begin{array}{c}\text { NITROGEN } \\
\text { Ascent/Descent }\end{array}$ & Not Available & Not Available & Not Available & & & Not available. \\
\hline $\begin{array}{l}\text { NITROGEN } \\
\text { On-Orbit }\end{array}$ & Not available. & None & None & $\begin{array}{l}12 \mathrm{lbm} / \mathrm{hr} \text { to rack and then to } \\
\text { payload. } \\
\text { No rack flow control. }\end{array}$ & & Not available. \\
\hline ACCESS & $\begin{array}{l}\text { ASCENT: L- } 18 \text { to } 24 \mathrm{hrs} \\
\text { DESCENT: } \mathrm{R}+3 \mathrm{hrs}\end{array}$ & $\begin{array}{l}\text { ASCENT: } L-40 \text { hrs (L-33 } \\
\text { hrs negotiable) } \\
\text { DESCENT: } R+5 \text { hrs }\end{array}$ & $\begin{array}{l}\text { ASCENT: L- } 88 \mathrm{hrs} \\
\text { DESCENT: R+96 hrs }\end{array}$ & & & $\begin{array}{l}\text { ASCENT: } \leq L-88 \text { hrs. } \\
\text { DESCENT: } \leq R+96 \\
\text { hrs }\end{array}$ \\
\hline $\begin{array}{l}\text { DEPRESS/ } \\
\text { REPRESS } \\
\text { RATES } \\
\text { (On-Orbit) }\end{array}$ & $\begin{array}{lc}\text { Depress: } & 24.0 \mathrm{psi} / \mathrm{min} . \\
\text { Repress: } & 9.0 \mathrm{psi} / \mathrm{min} .\end{array}$ & $\begin{array}{cc}\text { Depress: } & 9.0 \mathrm{psi} / \mathrm{min} . \\
\text { Repress: } & 0\end{array}$ & $\begin{array}{ll}\text { Depress: } & 7.75 \mathrm{psi} / \mathrm{min} . \\
\text { Repress: } & 6.96 \mathrm{ps} / \mathrm{min} .\end{array}$ & $\begin{array}{ll}\text { Depress: } & 7.64 \mathrm{psi} / \mathrm{min} . \\
\text { Repress: } & 2.00 \mathrm{psi} / \mathrm{min}\end{array}$ & & $\begin{array}{l}\text { Depress: } 24.0 \\
\text { psi/min. } \\
\text { Repress: } 9.0 \\
\text { psi/min. }\end{array}$ \\
\hline $\begin{array}{l}\text { ACOUSTIC } \\
\text { NOISE }\end{array}$ & $\begin{array}{c}\text { NSTS 2100-IDD-MDK, } \\
\text { Section 4.7- Environment } \\
\text { - ascent; Continuous PL } \\
\text { operation noise limits - on } \\
\text { orbit. } \\
\text { NASA Std 145A in NSTS } \\
\text { 08080-1 - Environment - } \\
\text { On-orbit; Intermittent } \\
\text { payload generated noise } \\
\text { limits. }\end{array}$ & $\begin{array}{c}\text { Per MIL-STD-1474D } \\
\text { Ref. MDC91W5023K (6.00) } \\
\text { Para. 4.4, 4.4.1 - ascent }\end{array}$ & $\begin{array}{l}\text { SSP52000-IDD-ERP, Para. 4.7.1 } \\
\text { \& Table 4-IX - Environment- } \\
\text { ascent. } \\
\text { No requirement for descent. }\end{array}$ & $\begin{array}{l}\text { Total Rack SPL: } \\
\text { SSP52000-IDD-ERP, Para. } \\
\text { 4.7.2 \& Fig. 4-2 } \\
\text { Rack Payload SPL: } \\
\text { SSP52000-IDD-ERP, Para. } \\
\text { 4.7.2.2 \& Table 4-X. }\end{array}$ & $\begin{array}{l}\text { Acoustic } \\
\text { Requirement for } \\
\text { payload operation on } \\
\text { ISS appears less } \\
\text { than SH or MD } \\
\text { operational } \\
\text { requirements, } \\
\text { therefore the ISS } \\
\text { requirement is the } \\
\text { controlling factor }\end{array}$ & $\begin{array}{l}\text { SSP52000-IDD-ERP, } \\
\text { Para. 4.7.2.2 \& Table } \\
\text { 4-X. }\end{array}$ \\
\hline $\begin{array}{c}\text { FIRE } \\
\text { PROTECTION }\end{array}$ & $\begin{array}{l}\text { PFE port required for actuve } \\
\text { payloads. }\end{array}$ & $\begin{array}{l}\text { Two smoke sensors in Air } \\
\text { Mixing Box. } \\
\text { Ten Halon Bottles (6\% vol. } \\
\text { Concentration at } 14.5 \text { psia) } \\
1 \text { hand held fire extinguisher } \\
2 \text { SEBs units }\end{array}$ & None, not powered. & $\begin{array}{l}\text { No PFE port required for } \\
\text { payloads that interface to the } \\
\text { rack AAA cooling loop. } \\
\text { Sealed container payloads do } \\
\text { not require a PFE port. }\end{array}$ & & $\begin{array}{l}\text { PFE port required for } \\
\text { active payloads. } \\
\text { MPLM- } \\
\text { No requirement, no } \\
\text { power. }\end{array}$ \\
\hline Materials & & & & & & $\begin{array}{l}\text { Envelope Worst Case } \\
\text { From All Carriers }\end{array}$ \\
\hline
\end{tabular}

